\title{
Is ultrasound-measured abdominal fat thickness a reliable method for predicting metabolic diseases in obese and overweight women?
}

\author{
Hooman Angoorani ${ }^{1}$, Zeinab Karimi ${ }^{1}$, Farokh Naderi ${ }^{2}$, Ali Mazaherinezhad ${ }^{* 3}$ \\ Received: 17 June 2017 \\ Published: 28 Aug 2018
}

\begin{abstract}
Background: Central fat deposition seems to be a risk factor for developing metabolic and cardiovascular diseases in overweight and obese individuals. Ultrasound is an accurate and non-invasive tool for measuring abdominal fat thickness and can precisely separate subcutaneous from visceral fat. This study was conducted to apply ultrasonography as a simple and reliable method to measure subcutaneous and visceral abdominal fat thickness and evaluate the relationship between this measured abdominal fat thickness and metabolic risk factors.

Methods: A total of 80 overweight and obese women were included in this study. Anthropometric indices and abdominal fat thickness were measured using ultrasound. The association between abdominal fat thickness and metabolic risk factors with anthropometric indices was assessed using correlation coefficient.

Results: The mean $( \pm$ SD) of subcutaneous and visceral fat thickness was $2.71 \pm 0.92$ and $5.46 \pm 1.88$, respectively. There was a relationship between waist circumference and visceral and subcutaneous fat thickness. Also, there was a relationship between ultrasoundmeasured visceral fat thickness and fasting blood glucose and triglyceride.

Conclusion: Ultrasonography is a simple and reliable method to measure abdominal fat thickness as an important predictor of metabolic diseases.
\end{abstract}

Keywords: Ultrasonography, Obesity, Visceral fat, Metabolic disease

Copyright $\odot$ Iran University of Medical Sciences

Cite this article as: Angoorani H, KarimiZ, Naderi F, Mazaherinezhad A. Is ultrasound-measured abdominal fat thickness a reliable method for predicting metabolic diseases in obese and overweight women? Med J Islam Repub Iran. 2018 (28 Aug);32:78. https://doi.org/10.14196/mjiri.32.78

\section{Introduction}

Obesity is a very common and rapidly growing metabolic disorder, which results in millions of annual deaths worldwide (1-7). Central fat deposition is undoubtedly a significant risk factor for metabolic and cardiovascular diseases in overweight and obese individuals $(8,9)$. Accumulation of visceral adipose tissue may result in insulin resistance; therefore, among different risk factors of metabolic diseases, intra-abdominal fat deposition has a critical role $(2,8)$. Some researchers found a relationship between the higher amount of abdominal fat with impaired fasting blood glucose, diabetes, hypertension, and metabolic syndrome (10). Undoubtedly, accurate measurement of abdominal fat is an essential part of every study, as it evaluates the risk factors for developing metabolic diseases ( 1 , $2,10)$.

\section{Corresponding author:Dr Ali Mazaherinezhad, mazaherinezhad@gmail.com}

1. Department of Sports and Exercise Medicine, Hazrat Rasool-e-Akram Hospital, Iran University of Medical Sciences, Tehran, Iran.

2. Department of Radiology, Hazrat Rasool-e-Akram Hospital, Iran University of Medical Sciences, Tehran, Iran.

3. Minimally Invasive Surgery Research Center, Iran University of Medical Sciences, Tehran, Iran.
Anthropometric parameters, such as body mass index (BMI), cannot distinguish subcutaneous fat from visceral fat accurately, so these parameters cannot predict the risk of metabolic and cardiovascular diseases independently $(1,11)$. It has been proposed that CT scan and MRI provide a good estimation of abdominal visceral fat thickness $(12,13)$. However, exposure to high doses of radiation, high cost, and low availability make applying this procedure unreasonable in routine medical practice. It has been shown that ultrasound is an accurate, non-invasive, and reliable method to estimate intra-abdominal and visceral fat $(2,8,12-17)$. Moreover, the accuracy of ultrasound in assessing visceral fat seems to be as high as CT scan (13).

Although subcutaneous and visceral fat are both considered as metabolic risk factors, visceral fat has a stronger

$\uparrow$ What is "already known” in this topic:

Central fat deposition is an important risk factor for developing metabolic and cardiovascular diseases.

\section{$\rightarrow$ What this article adds:}

In comparison with anthropometric indices, ultrasonography is a more reliable and accurate method to measure abdominal visceral fat thickness. 
relationship with metabolic syndrome (10, 18-20). There are limited data displaying that ultrasound-measured abdominal fat thickness, especially in women, is more related to metabolic risk factors than usual anthropometric indices $(9,10)$. To our knowledge, no study in Iran has evaluated the association between ultrasound-measured abdominal fat thickness and metabolic risk factors. Thus, the present study was conducted to assess whether ultrasound-measured abdominal fat thickness is a reliable method for predicting metabolic risk factors in obese and overweight Iranian women.

\section{Methods}

\section{Data}

This case series study was the first survey in Iran to evaluate the relationship between ultrasound-measured abdominal fat thickness and metabolic risk factors in Iranian overweight and obese women.

A total of 80 overweight and obese women, who visited the obesity center of Rasool-e-Akram hospital in Tehran, were included in this study.

Inclusion criteria were as follow: women aged 18 to 65 years; BMI of 25 to 40; absence of pregnancy and lactation; lack of history of uncontrolled endocrine diseases, cardiovascular diseases, and bleeding disorders; no history of consumption of weight loss medications or supplements within the last 3 months; and no history of applying other weight loss methods.

Exclusion criteria were as follow: any weight loss surgery during the study, use of corticosteroids or contraceptive medications, and patient's request for leaving the study.

\section{Codes of ethics}

All participants were given information about the purpose of the study and asked to sign an informed consent before the study.

This study was a part of a clinical trial (registration code: IRCT2014217858) and was approved by Ethics Committee of Iran University of Medical Sciences (code: 99888).

\section{Measurement of the anthropometric indices}

Weight, height, waist, and hip circumferences were measured as the anthropometric indices in this study. These measurements were repeated 3 times, and if the difference between the 2 measurements was more than 5 $\mathrm{mm}$, it was repeated again.

Weight assessment: Weight measurement was performed for all samples using SECA model scale with \pm 50 grams accuracy, while the participants were requested to have lightweight clothes on during the weighing process. Approximately, 500 grams was considered for the weight of the remaining clothes. All measurements were performed at 9 to $11 \mathrm{AM}$, while the samples were fasted, and all patients were asked to empty their bladder before weighing.

Height assessment: All patients were asked to take off their shoes, socks, and any head accessories; then, they were asked to stand against the wall with their feet togeth- er.

Patients' height was measured using 200 centimeters length stadiometer, which was fixed on the wall.

Body mass index (BMI): Body mass index (BMI) is a person's weight in kilograms divided by the square of height in meters. If BMI was 25.0 to 29.9, it fell within the overweight range, and if BMI was 30.0 or higher, it fell within the obese range.

Waist-hip ratio: Waist-hip ratio is the ratio of the circumference of the waist to that of the hip. This is calculated as waist measurement divided by hip measurement. Waist circumference was measured at the midpoint (a place with the lowest diameter) between umbilicus to the bottom of the xyphoid using a stretch-resistant tape. Hip circumference was measured around the widest part of the buttocks, with the tape parallel to the floor. The measurements were repeated twice in each case, and if the difference between the 2 measurements was not more than 5 $\mathrm{mm}$, the average number was then recorded.

\section{Abdominal fat measurement}

Abdominal fat was measured using ultrasound (Voluson 730 PRO model; GE Company). All measurements were performed at 8 to 9 AM with the same device. All measurements were based on millimeter scale and done by merely 1 radiologist with more than 10 years of experience in the field of musculoskeletal ultrasonography. Both subcutaneous and visceral fat was measured during the sonographic assessment. To improve the accuracy of measurements, all samples were asked to be fasted before the examination. During the examination, patients were asked to take a deep inspiration, followed by a deep expiration, and then keep their breath for a few seconds till the sonographer could fix the image during the measurements.

\section{Measurement of the abdominal subcutaneous fat with} ultrasound

Linear probe with frequency of 6-8 $\mathrm{Hz}$ was applied to measure abdominal subcutaneous fat, while the probe was kept in the transverse position. Main land mark was umbilicus. The probe was placed slightly higher than upper edge of the umbilicus. The distance between underderm to the external fascia of the abdominal rectus muscle was measured. The measurement was repeated 3 times and the average value was recorded (Fig. 1).

\section{Measurement of the abdominal visceral fat with ultra-} sound

Curve probe with frequency of $2-7 \mathrm{~Hz}$ was applied to measure abdominal visceral fat, while the probe was kept in the longitudinal position. To determine the best place for the measurement, umbilicus was found first and, then, the probe was placed slightly higher than the upper edge of umbilicus. After detecting the lumbar vertebra, which is located at the back of aortic artery, vertical distance between the internal fascia of the abdominal rectus muscle and the anterior wall of the vertebra was measured. The measurement was repeated 3 times and the average value was recorded (Fig. 2). 


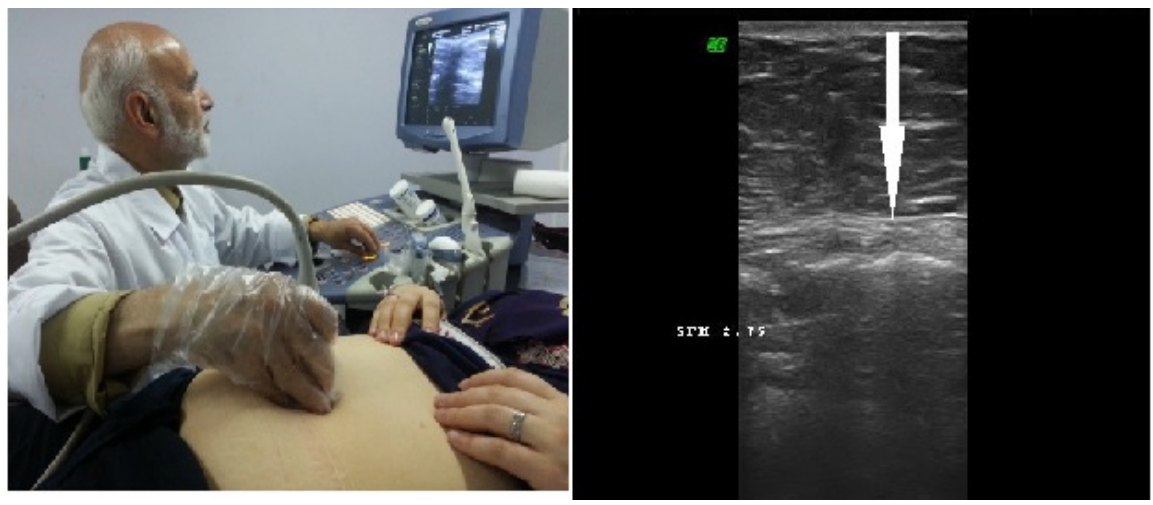

Fig. 1. Measurement of Subcutaneous abdominal fat with ultrasound

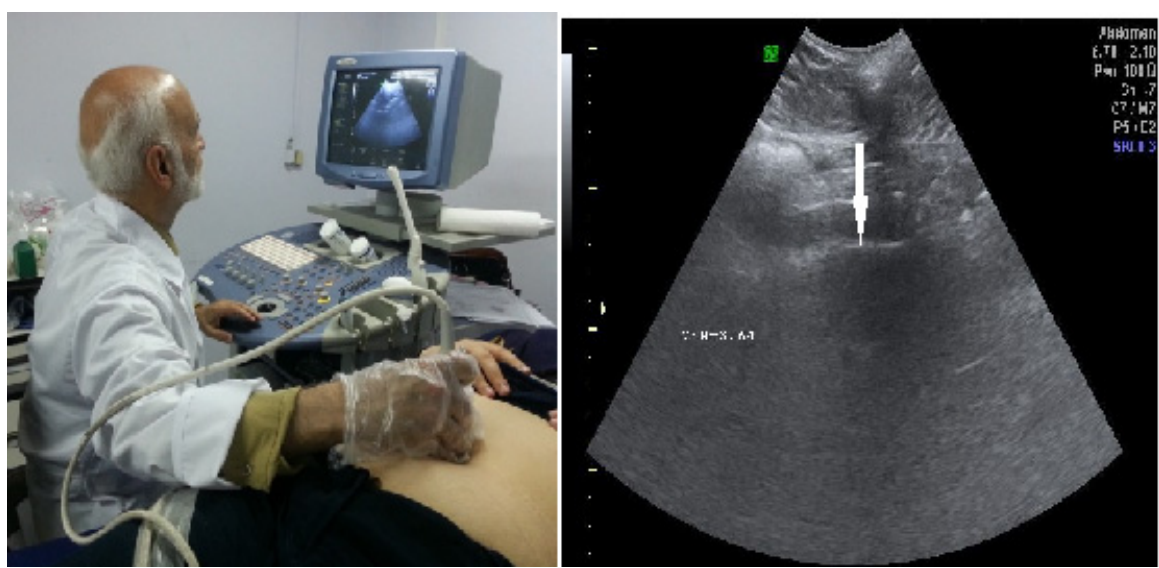

Fig. 2. Measurement of visceral abdominal fat with ultrasound

\section{Metabolic risk factors}

In addition to anthropometric indices, some laboratory tests, including fasting blood glucose, total blood cholesterol, high density lipoprotein (HDL), and low-density lipoprotein (LDL) of serum were considered as metabolic risk factors in this study. For this purpose, patients were asked to fast for at least 12 hours and, then, peripheral blood samples were taken from the antecubital vein using vacuum tubes Greiner. Samples were analyzed immediately and blood glucose and lipid profile were checked.

\section{Outcome measurement}

Primary outcome: The primary outcome of the present study was evaluation of the relationship between ultrasound-measured abdominal visceral fat thickness and metabolic risk factors in Iranian women.

Secondary outcome: The secondary outcome of the study was evaluation of the relationship between subcuta- neous and visceral fat measured by ultrasound and anthropometric indices and also evaluation of the relationship between anthropometric indices and metabolic risk factors in Iranian women.

\section{Statistical analysis}

SPSS Version 22 was used for data analysis. Mean and standard deviation, Kolmogorov-Smirnov test, and Pearson correlation coefficient were used to describe the variables, normality of variables, and intervariable relationship, respectively. Significance level was set at $p<0.05$.

\section{Results}

A total of 80 women aged 21 to 66 years participated in this study. Table 1 displays the demographic characteristics of the participants.

The mean $( \pm \mathrm{SD})$ of subcutaneous fat measured by ultrasonography was $2.71 \pm 0.92$, and the mean $( \pm \mathrm{SD})$ of

\begin{tabular}{lcc} 
Table 1. Demographic characteristics of the study population & \\
\hline Variable & Mean \pm SD & Range \\
\hline Age (year) & $46.93 \pm 10.01$ & $21-66$ \\
Weight (Kg) & $79.77 \pm 11.58$ & $62.10-123.10$ \\
Height (cm) & $160.19 \pm 6.77$ & $144-178$ \\
BMI & $31.02 \pm 3.43$ & $24.59-39.29$ \\
Waist circumference (cm) & $90.52 \pm 8.53$ & $70-114.40$ \\
Waist to hip ratio & $0.82 \pm 0.06$ & $0.67-0.98$ \\
Hip circumference & $109.93 \pm 7.64$ & $95-131$ \\
FBS & $94.33 \pm 15.30$ & $69-144$ \\
TG & $131.86 \pm 59.79$ & $49-317$ \\
Total cholesterol & $197.43 \pm 41.33$ & $107-311$ \\
HDL & $49.97 \pm 12.26$ & $31-82$ \\
\hline
\end{tabular}


Table 2. Pearson correlation between subcutaneous and visceral fat measured by ultrasound with the anthropometric indexes

\begin{tabular}{lcccc}
\hline Variable & \multicolumn{3}{c}{ Subcutaneous fat } & \multicolumn{2}{c}{ Visceral fat } \\
\cline { 2 - 5 } & $\mathrm{r}$ & $\mathrm{p}$ & $\mathrm{r}$ & \\
Waist circumference & 0.24 & $0.02^{*}$ & 0.6 & $<0.0001^{*}$ \\
Hip circumference & 0.26 & $0.01^{*}$ & 0.1 & 0.34 \\
Waist to hip ratio & 0.06 & 0.58 & 0.59 & $<0.0001^{*}$ \\
\hline
\end{tabular}

Table 3. Correlation between intraabdominal fat and waist circumference with metabolic risk factors

\begin{tabular}{|c|c|c|c|c|c|c|c|c|c|c|}
\hline & $\begin{array}{c}\text { Waist } \\
\text { circumference }\end{array}$ & $\mathrm{p}$ & $\begin{array}{l}\text { subcutaneous } \\
\text { fat by US }\end{array}$ & $\mathrm{p}$ & $\begin{array}{l}\text { visceral } \\
\text { fat by US }\end{array}$ & $\mathrm{p}$ & $\begin{array}{c}\text { Hip } \\
\text { circumference }\end{array}$ & $\mathrm{p}$ & WHR & $\mathrm{p}$ \\
\hline BMI & 0.78 & $<0.0001^{*}$ & 0.42 & $<0.0001^{*}$ & 0.36 & $0.001 *$ & 0.72 & $<0.0001^{*}$ & 0.29 & $0.009^{*}$ \\
\hline FBS & 0.264 & $0.018^{*}$ & 0.006 & 0.95 & 0.39 & $0.001 *$ & -0.08 & 0.46 & 0.37 & $0.001 *$ \\
\hline TG & 0.331 & $0.003 *$ & 0.15 & 0.18 & 0.36 & $0.001 *$ & -0.02 & 0.82 & 0.38 & $<0.0001 *$ \\
\hline $\begin{array}{l}\text { Total } \\
\text { cholesterol }\end{array}$ & 0.127 & 0.263 & -0.08 & 0.47 & 0.20 & 0.06 & 0 & 0.99 & 0.14 & 0.19 \\
\hline HDL & 0.095 & 0.401 & -0.065 & 0.56 & 0.12 & 0.28 & -0.004 & 0.97 & 0.11 & 0.31 \\
\hline LDL & 0.074 & 0.516 & -0.21 & 0.06 & 0.16 & 0.13 & -0.001 & 0.99 & 0.08 & 0.43 \\
\hline
\end{tabular}

visceral fat measured by ultrasonography was $5.46 \pm 1.88$. The visceral fat measured by ultrasonography was weakly correlated with age $(\mathrm{r}=0.32: \mathrm{p}=0.003)$, but this correlation was not observed between ultrasound-measured subcutaneous fat and age $(\mathrm{r}=-0.16: \mathrm{p}=0.13)$. Waist circumference and waist to hip ratio were also weakly correlated with age (orderly: $\mathrm{r}=0.33: \mathrm{p}=0.003, \mathrm{r}=0.41: \mathrm{p}<0.0001$ ), but this correlation was not observed between hip circumference and age $(\mathrm{r}=-0.03: \mathrm{P}=0.73)$. Also, there was no association between age and BMI $(r=0.14: p=0.19)$.

There was a correlation between waist and hip circumferences and ultrasound-measured abdominal subcutaneous fat $(\mathrm{p}<0.05$, Table 2$)$. Also, there was a correlation between waist circumference and waist to hip ratio and abdominal visceral fat ( $\mathrm{p}<0.0001$, Table 2, Fig. 3$)$. There was a correlation between ultrasound-measured subcutaneous fat thickness and BMI ( $p<0.0001$, Table 3$)$. Moreover, there was a correlation between ultrasoundmeasured visceral fat thickness and some metabolic risk factors, including fasting blood glucose, triglyceride, and BMI ( $<<0.001$, Table 3, Fig. 4).

\section{Discussion}

This study revealed that ultrasound-measured abdominal visceral fat thickness can be considered as an important predictor of metabolic diseases in Iranian women.

In most of previous studies, waist circumference and waist to hip ratio have been the most frequently used anthropometric indices for estimation of intra-abdominal fat $(2,9)$. Waist circumference and waist to hip ratio seem to be reliable methods to estimate the level of fat deposition in the trunk and abdomen area $(2,16,17)$. However, very few studies have evaluated the relationship among anthropometric indices, ultrasound-measured subcutaneous and visceral fat thickness.

According to this study, there were correlations between ultrasound-measured subcutaneous and visceral fat thickness and some parameters of anthropometric indices. This study revealed that ultrasound-measured subcutaneous fat thickness is related to waist and hip circumferences, while ultrasound-measured visceral fat thickness is more related to waist circumference and waist to hip ratio. Moreover, anthropometric indices are more correlated with ultra- sound-measured visceral fat thickness than subcutaneous fat thickness $(\mathrm{r}=0.6 ; \mathrm{p}<0.0001$ vs $\mathrm{r}=0.24 ; \mathrm{p}<0.02)$.

Since anthropometric indices cannot accurately distinguish subcutaneous fat from visceral fat, some researchers considered some imaging techniques, such as CT scan, MRI, and particularly ultrasonography, for more accurate measurement of visceral fat as a main predictor of metabolic diseases $(2,10-15)$. This study demonstrated that ultrasound-measured visceral fat thickness is strongly related to some metabolic risk factors, including fasting blood glucose, triglyceride, and BMI. This finding is in

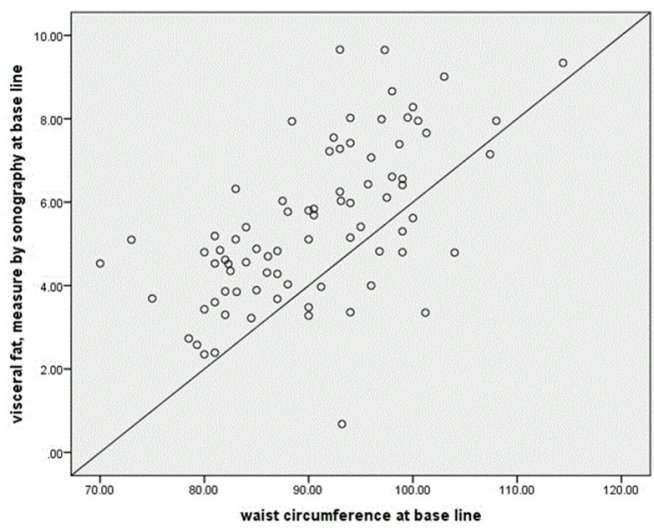

Fig. 3. Correlation between visceral abdominal fat with waist circumference

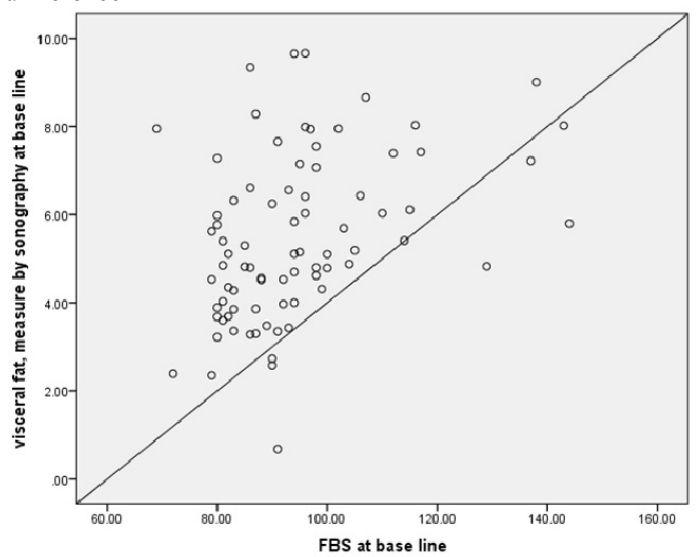

Fig. 4. Correlation between visceral abdominal fat with fasting blood glucose 
accordance with the results of several previous surveys (Bjorntorp et al., Fujioka et al., Taksali et al., and Leite et al.) that found abdominal visceral fat thickness is related to metabolic and cardiovascular risk factors (19-22).

This study, similar to previous surveys (23-25), revealed that ultrasound- measured abdominal visceral fat thickness is a better predictor of metabolic diseases than the routinely used anthropometric indices, such as BMI or waist and hip circumferences.

Based on this study, neither ultrasound- measured abdominal fat thickness, nor anthropometric indices were correlated with the blood level of total cholesterol, lowdensity lipoprotein (LDL), and high-density lipoprotein (HDL). This finding contradicts the result of the study of Leite et al., (22) who reported that abdominal visceral fat thickness measured by ultrasound is related to metabolic risk factors, including blood lipid profile. However, this difference can be due to using different criteria for defining abnormal level of blood cholesterol in this study compared to Leite's study.

In contrast to many previous studies and similar the study of Meeuwsen et al. (26), this study found a moderate correlation between BMI and abdominal subcutaneous fat thickness measured by ultrasound, which might be due to ethnic or racial differences.

\section{Limitations and Strengths}

This study had some limitations. First, although case series studies such as this one are useful and efficient in obtaining general information about a target population, they may not be able to assess causality. Moreover, in such studies, sample sizes may not be large enough to detect all relations. Second, since all participants were female, this study was not able to determine the effect of gender differences. Finally, all participants had to undergo a special preparation for sonographic evaluation, which may not be possible in a routine medical practice.

In this study, ultrasound was applied to measure abdominal fat thickness, which is a highly accurate method. Moreover, all sonographic evaluations were performed only by an experienced radiologist, who made the measurements more reliable.

\section{Conclusion}

The present study found that, compared to anthropometric indices, ultrasonography is a more reliable and accurate method to measure abdominal visceral fat thickness as an important predictor of metabolic diseases.

\section{Acknowledgment}

Authors gratefully acknowledge the Obesity and Minimally Invasive Surgery Research Center of Hazrat Rasoole-Akram hospital for its valuable support.

\section{Source of funding \\ Iran University of Medical Sciences}

\section{Conflict of Interests}

The authors declare that they have no competing interests.

\section{References}

1. Rolfe EDL, Sleigh A, Finucane FM, Brage S, Stolk RP, Cooper C, et al. Ultrasound measurements of visceral and subcutaneous abdominal thickness to predict abdominal adiposity among older men and women. Obesity. 2010;18(3):625-31

2. Ribeiro-Filho FF, Faria AN, Azjen S, Zanella MT, Ferreira SR Methods of estimation of visceral fat: advantages of ultrasonography Obesity Res. 2003;11(12):1488-94.

3. Dabbaghmanesh MH. Effects of body electroacupuncture on plasma leptin concentrations in obese and overweight people in Iran: a randomized controlled trial. Altern Ther Health Med.2013;19(2):24.

4. Rahmani A, Sayehmiri K, Asadollahi K, Sarokhani D, Islami F, Sarokhani M. Investigation of the Prevalence of Obesity in Iran: a Systematic Review and Meta-Analysis Study. Acta Medica Iranica. 2015;53(10):596-607.

5. world health organization. Obesity and overweight: world health organization; 2015 [cited 2016 2016.06.03]. Available from: $\mathrm{http} / / / \mathrm{www}$. who.int/mediacentre/factsheets/fs311/en/.

6. Nojomi M, Moradi-Lakeh M, Velayati A, Naghibzadeh-Tahami A, Dadgostar H, Ghorabi G, et al. Health technology assessment of noninvasive interventions for weight loss and body shape in Iran. Med J Islam Rep Iran. 2016;30(1):327-40.

7. Kiadaliri AA, Jafari M, Mahdavi MRV, Faghihzadeh S, Kalantari N, Asadi-Lari M. The prevalence of adulthood overweight and obesity in Tehran: findings from Urban HEART-2 study. Med J Islam Rep Iran. 2015;29:178.

8. Stolk R, Wink O, Zelissen P, Meijer R, Van Gils A, Grobbee D. Validity and reproducibility of ultrasonography for the measurement of intra-abdominal adipose tissue. Int J Obes (Lond). 2001;25(9).

9. Stolk RP, Meijer R, Mali WP, Grobbee DE, van der Graaf Y. Ultrasound measurements of intraabdominal fat estimate the metabolic syndrome better than do measurements of waist circumference. Am. J. Clin. Nutr. 2003;77(4):857-60.

10. Fox CS, Massaro JM, Hoffmann U, Pou KM, Maurovich-Horvat P, Liu CY, et al. Abdominal visceral and subcutaneous adipose tissue compartments association with metabolic risk factors in the Framingham Heart Study. Circulation. 2007;116(1):39-48.

11. Schreiner PJ, Terry JG, Evans GW, Hinson WH, Crouse JR, Heiss G. Sex-specific associations of magnetic resonance imaging-derived intra-abdominal and subcutaneous fat areas with conventional anthropometric indices the atherosclerosis risk in communities study. Am. J. Epidemiol. 1996;144(4):335-45.

12. Ribeiro-Filho FF, Faria AN, Kohlmann O, Ajzen S, Ribeiro AB, Zanella MT, et al. Ultrasonography for the evaluation of visceral fat and cardiovascular risk. Hypertension. 2001;38(3):713-7.

13. Hirooka M, Kumagi T, Kurose K, Nakanishi S, Michitaka K, Matsuura B, et al. A technique for the measurement of visceral fat by ultrasonography: comparison of measurements by ultrasonography and computed tomography. Internal Med. 2005;44(8):794-9.

14. Ribeiro-Filho FF, Faria AN, Kohlmann OJr, Ajzen S, Ribeiro AB, Zanella MT, et al. Ultrasonography for the evaluation of visceral fat and cardiovascular risk. Hypertension. 2001;38(3 Pt 2):713-7.

15. van der Kooy K, Seidell JC. Techniques for the measurement of visceral fat: a practical guide. Int J Obes (Lond). 1993;17(4):187-96.

16. Pouliot MC, Després JP, Lemieux S, Moorjani S, Bouchard C, Tremblay A, et al. Waist circumference and abdominal sagittal diameter: best simple anthropometric indexes of abdominal visceral adipose tissue accumulation and related cardiovascular risk in men and women. Am. J. Cardiol. 1994;73(7):460-8.

17. Han T, McNeill G, Seidell J, Lean M. Predicting intra-abdominal fatness from anthropometric measures: the influence of stature. Int $\mathrm{J}$ Obes (Lond). 1997;21(7):587-93.

18. DeFronzo RA, Ferrannini E. Insulin resistance: a multifaceted syndrome responsible for NIDDM, obesity, hypertension, dyslipidemia, and atherosclerotic cardiovascular disease. Diabetes care. 1991;14(3):173-94.

19.Bjorntorp P. "Portal" adipose tissue as a generator of risk factors for cardiovascular disease and diabetes. Arteriosclerosis (Dallas, Tex). 1990;10(4):493-6.

20. Fujioka S, Matsuzawa Y, Tokunaga K, Tarui S. Contribution of intra-abdominal fat accumulation to the impairment of glucose and lipid metabolism in human obesity. Metabolism. 1987;36(1):54-9.

21. Taksali SE, Caprio S, Dziura J, Dufour S, Calí AM, Goodman TR, et al. High visceral and low abdominal subcutaneous fat stores in the 
obese adolescent A determinant of an adverse metabolic phenotype Diabetes. 2008;57(2):367-71

22. Leite CC, Wajchenberg BL, Radominski R, Matsuda D, Cerri GG, Halpern A. Intra-abdominal thickness by ultrasonography to predict risk factors for cardiovascular disease and its correlation with anthropometric measurements. Metabolism. 2002;51(8):1034-40.

23. Amati F, Pennant M, Azuma K, Dubé JJ, Toledo FG, Rossi AP, et al. Lower thigh subcutaneous and higher visceral abdominal adipose tissue content both contribute to insulin resistance. Obesity. 2012;20(5):1115-7.

24. Medina-Urrutia A, Posadas-Romero C, Posadas-Sánchez R, JorgeGalarza E, Villarreal-Molina T, Gonzalez-Salazar Mdel C, et al. Role of adiponectin and free fatty acids on the association between abdominal visceral fat and insulin resistance. Cardiovasc Diabetol. 2015;14:20.

25. Messier V, Karelis AD, Prud'homme D, Primeau V, Brochu M, Rabasa-Lhoret R. Identifying metabolically healthy but obese individuals in sedentary postmenopausal women. Obesity (Silver Spring, Md). 2010;18(5):911-7.

26. Meeuwsen S, Horgan G, Elia M. The relationship between BMI and percent body fat, measured by bioelectrical impedance, in a large adult sample is curvilinear and influenced by age and sex. Clin Nutr. 2010;29(5):560-6. 